\title{
A Comparable Study On ZnO-Based Room Temperature Ozone Sensing Characteristics Utilizing Conductometric and Surface Acoustic Waves Techniques
}

\author{
Katerinopoulou $\mathrm{D}^{1,2 \star}$, Moschovis $\mathrm{K}^{1,2}$, Gagaoudakis E1,2, Aperathitis E2, Binas $\mathrm{V}^{1-3}$ and Kiriakidis $\mathrm{G}^{1-3}$ \\ IDepartment of Physics, University of Crete, P.O. Box 2208, 71003 Heraklion, Crete, Greece \\ 2Institute of Electronic Structure and Laser (IESL), Foundation for Research and Technology Hellas (FORTH), PO Box 1385, Vasilika Vouton, \\ 70013 Heraklion, Crete, Greece \\ ${ }^{3}$ Crete Center for Quantum Complexity and Nanotechnology, Department of Physics, University of Crete, P.O. Box 2208, 71003 Heraklion, Greece
}

\section{Article Info}

*Corresponding author:
D. Katerinopoulou
Department of Physics
University of Crete
P.O. Box 2208, 71003 Heraklion
Crete, Greece
E-mail: dkaterin@iesl.forth.gr

Received: March 9, 2017

Accepted: April 3, 2017

Published: April 10, 2017

Citation: Katerinopoulou D, Moschovis K, Gagaoudakis E, Aperathitis E, Binas V, Kiriakidis G. A Comparable Study on ZnO based Room Temperature Ozone Sensing Characteristics utilizing Conductometric and Surface Acoustic Waves Techniques. Madridge J Nanotechnol Nanosci. 2017; 2(1): 44-51. doi: 10.18689/mjnn-1000109

Copyright: @ 2017 The Author(s). This work is licensed under a Creative Commons Attribution 4.0 International License, which permits unrestricted use, distribution, and reproduction in any medium, provided the original work is properly cited.

Published by Madridge Publishers

\begin{abstract}
Ozone $\left(\mathrm{O}_{3}\right)$ detection is of great interest for public safety and human health. In this work, zinc oxide $(\mathrm{ZnO})$ films were tested as sensing elements against different ozone concentrations. The DC Magnetron Sputtering technique was employed to deposit $\mathrm{ZnO}$ films of various thicknesses on both glass as well as commercial transducers, at room temperature. Surface, structural and optical properties were studied by Atomic Force Microscopy, X-Ray Diffraction and spectrophotometrytechniques, respectively, revealing that ZnO films had a polycrystalline structure, exhibiting a visible transmittance over $80 \%$ and an energy gap of about $3.31 \mathrm{eV}$. The sensing properties of films were investigated by employing both the conductometric and Surface Acoustic Waves (SAW) techniques. Utilizing the first technique, the films exhibited a room temperature (RT) response of five orders of magnitude under an ultra-low ozone concentration of 5 parts per billion ( $\mathrm{ppb}$ ), while applying the SAW technique, utilizing SAW filters operating at a nominal frequency of $930 \mathrm{MHz}$ a response of a few $\mathrm{MHz}$ was recorded for ozone concentrations of the order of $100 \mathrm{ppb}$. Films tested under both techniques were found to have fast response rates and good reversibility, which merits them for room temperature durable ozone sensing device applications.
\end{abstract}

Keywords: DC sputtering; ZnO films; Ozone detection at RT; Conductometric Sensors; SAW sensors.

\section{Introduction}

Zinc oxide $(\mathrm{ZnO})$ belongs to the II - IV category of semiconductors with a wide direct energy band gap of $3.32 \mathrm{eV}$ at room temperature [1]. Advantages associated with its wide band gap include high breakdown voltages, ability to sustain large electric fields, low electronic noise and high-temperature / high-power operation [2]. ZnO is an intrinsic n-type semiconductor [2-4] due to oxygen vacancies or zinc interstitials and crystallizes in two main structures, wurtzite of hexagonal phase and zinc blende of cubic phase with tetrahedral coordination geometry in both cases [5-7]. It is also one of the preferable metal oxide materials (together with In and $\mathrm{Sn}$ ) for gas sensing applications, due to its high sensitivity [8], high stability, perfect c-axis orientation texture and piezoelectric properties. Based on these, $\mathrm{ZnO}$ has allowed for the development of surface acoustic wave (SAW) sensing devices that can operate in a wide range of frequencies [9]. Therefore, a significant part of the recent research in the field of $\mathrm{ZnO}$-based sensing devices and applications deals with $\mathrm{ZnO}$ 
nanostructures and their integration with mainstream semiconductor materials [10]. Ozone is an important component of smog and a highly reactive and unstable gas capable of damaging living cells. According to the U. S. Environmental Protection Agency (EPA) [11], ozone concentrations of more than 70 ppb are very dangerous for human health. Consequently, a need for the development of gas sensors, to improve the detection limits has emerged. Based on these, a number of groups have reported on the ozone sensing properties of various materials, such as $\ln _{2} \mathrm{O}_{3}$ prepared by sol-gel and RF sputtering techniques [12], $\mathrm{CuAlO}_{2}$ prepared by wet chemical route operating at temperature of $200^{\circ} \mathrm{C}$ [13], IGZO thin films deposited by RF magnetron sputtering [14] and $\mathrm{ZnO}$ grown by molecular beam epitaxy operating at room temperature [15]. However, the majority of these studies were focused on the investigation of sensing properties based on the material transport properties, utilizing conventional homemade or commercial transducers and only a limited number have applied techniques such as Surface Acoustic Waves (SAWs) [9].

Acoustic wave devices, as sensors have a potential range of applications due to the fact that they are small in size, relatively inexpensive and highly sensitive to surface perturbations that affect the velocity and the amplitude of the wave that propagates on the surface of the material [16] [17] [18]. The coupling between strain and electrical polarization that occurs in piezoelectric crystals provides the means for generating acoustic waves electrically. When a crystal structure lacks a centre of inversion symmetry, the application of strain changes the distribution of charge on the atoms and bonds comprising the crystal in such a manner that a net, macroscopic, electrical polarization of the crystal is created. Crystals exhibiting this direct piezoelectric effect always exhibit the converse effect as well, whenever the crystal is strained by the application of an electric field [19].

In this work, a comparable study of sputtered $\mathrm{ZnO}$ thin film sensing characteristics operating at $\mathrm{RT}$ is reported utilizing a conventional simple conductometric technique and SAW filter transducers both of which present potential alternative to conventional «Taguchi sensor» set-ups operating at elevated temperatures. Although previous studies [3] [4] [9] have shown the high sensitivity of metal oxides on detecting ultra-low ozone concentrations at the ppb level, this is the first time that a comparable study on the same material $(\mathrm{ZnO})$ is made in a direct effort to promote advantages and reveal weaknesses between the conductometric and SAW based techniques for gas $\left(\mathrm{O}_{3}\right)$ sensing applications.

\section{Experimental Details}

\section{Deposition technique}

The deposition of $\mathrm{ZnO}$ thin films was carried out in an Alcatel DC magnetron sputtering system using a $15 \mathrm{~cm}$ diameter, 99.999\% pure metallic zinc target, at room temperature. The base pressure prior to deposition was under $3 \times 10^{-6} \mathrm{mbar}$, while during deposition was kept constant at $8 \times 10^{-3} \mathrm{mbar}$. The plasma current and voltage settings were $0.25 \mathrm{~A}$ and $280 \mathrm{~V}$, respectively and the sputtering gas was $100 \%$ oxygen. Films were deposited onto Corning 1737F glass with $(25.4 \pm 0.2) \mathrm{mm} \times(25.4 \pm 0.2) \mathrm{mm}$ dimensions and a thickness of $(0.70 \pm 0.07) \mathrm{mm}$. The glasses were initially cleaned under sonication for $5 \mathrm{~min}$ with acetone and $5 \mathrm{~min}$ with isopropanol, then washed with distilled water and finally dried by $\mathrm{N}_{2}$. Then, $\mathrm{NiCr}$ Ohmic contacts were thermally evaporated at a pressure less than $10^{-6} \mathrm{mbar}$, for conductivity measurements. The thickness of the films was determined by using a profilometer (Veeco Dektak 150) and the step was formed by employing Aluminum tape on glass during deposition. $\mathrm{ZnO}$ films were also deposited on high frequency $(930 \mathrm{MHz})$ commercial SAW filters (Colledge Electronics Ltd), under the same deposition parameters.

\section{Sample characterization}

The surface morphology was studied via Atomic Force Microscopy (AFM) using a nasoscope IIIA microscope, in tapping mode with Si tip and resonant frequency $350 \mathrm{kHz}$. The crystallinity of the structure was determined by X-Ray Diffraction (XRD) technique using a Rigaku RINT 2000 diffractometer with CuKa X-Rays in a conventional goniometer employing $\theta-2 \theta$ scanning method. From the XRD pattern the crystallite size was calculated using Scherrer's formula [20]:

$$
D=\frac{0.9 \lambda}{\beta \cos \theta}
$$

where, $\lambda=0.154 \mathrm{~nm}$ is the $X$-Ray wavelength, $\theta$ is the Bragg angle and $\beta$ is the line broadening at half the maximum intensity (FWHM) in radians. The $\mathrm{d}$-spacing was calculated by Bragg's equation $\lambda=2 d \sin \theta$, where $d$ is the interplanar spacing and $\theta$ the diffraction angle [21].

The optical study was carried out through transmittance measurements, using a Perkin Elmer Lambda 950 spectrophotometer at a wavelength range of $\lambda=250-2500 \mathrm{~nm}$. From the transmittance spectrum and the equation $\mathrm{T}=\mathrm{T}_{0} \cdot \exp (\mathrm{ad})$ where $\mathrm{T}$ is the measured transmittance, $T_{0}$ is the glass transmittance (without material), $a$ is the absorption coefficient and $d$ the thickness of the films, the optical energy band gap $\left(E_{\text {gap }}\right)$ was evaluated by applying a Tauc plot, using the equation:

$$
(a h v)^{n}=A\left(h v-E_{\text {gap }}\right)
$$

where, $\alpha$ is the absorption coefficient, hv is the photon energy, $\mathrm{h}$ is Planck's constant and $\mathrm{A}$ is a constant. For a direct allowed optical transition, as for $\mathrm{ZnO}$ in the present study, $\mathrm{n}$ equals 2. From the plot of $(a h v)^{2}$ vs hv and by linear extrapolation, the energy gap was extracted.

\section{Sensing characterization techniques}

The ozone sensing properties of the films were estimated by monitoring electrical and SAW signals respectively at room temperature. Specifically, the conductometric measurements were carried out in a home-made system described elsewhere utilizing a consecutive photoreduction and oxidation process [22]. The photoreduction took place at a vacuum pressure of approximately 10-2 mbar while the samples were directly irradiated by a UV light (mercury pencil lamp with an average intensity of $4 \mathrm{~mW} / \mathrm{cm}^{2}$ ) at a wavelength of $254 \mathrm{~nm}$, placed at a distance of approximately $3 \mathrm{~cm}$ from the surface of the film. For the subsequent oxidation process ozone was produced by an ozone analyzer (Thermo Electron Corporation, model 49i) at different concentrations ranging between 5 and $2400 \mathrm{ppb}$ and the chamber was backfilled with 
ozone at a pressure of $100 \mathrm{mbar}$. In order to measure the induced electrical changes on the films during the photoreduction-oxidation procedure, a constant voltage was applied during the whole cycling procedure and the electric current was measured by a Keithley 6517A electrometer. In addition, the SAW filter tests took place in a corresponding home-made cell of approximately $30 \mathrm{ml}$ in volume. The cell incorporated a UV pencil style lamp fitting together with a quartz glass located directly opposite the filter structure and it was open to gas flow through inlet and outlet fittings while a labview based program was developed to facilitate automatic computer calculations. ZnO films were deposited onto commercial SAW filters and the whole structure exposed to the same photoreduction and subsequent oxidation processes. The $\mathrm{O}_{3}$ gas concentrations were ranging between $100 \mathrm{ppb}$ and $2 \mathrm{ppm}$ accurately produced by the ozone analyzer. The gas flow rate was kept at 500 standard $\mathrm{cm}^{3}$ / $\mathrm{min}(\mathrm{sccm})$ for both processes during all test cycles.

\section{Results and Discussion}

\section{Surface, structural and optical properties}

AFM surface characterization of the films showed that all films exhibited granular morphology without any cracks (Fig. 1). Furthermore, both the lateral grain size and the RMS roughness were found to increase with film thickness (Table 1), in accordance to the literature [23].

Table 1: Structural and optical properties of sputtered ZnO films

\begin{tabular}{ccccc}
\hline $\begin{array}{c}\text { Thickness } \\
(\mathrm{nm})\end{array}$ & $\begin{array}{c}\text { Optical band gap } \\
\mathrm{E}_{\text {gap }}(\mathrm{eV})\end{array}$ & $\begin{array}{c}\text { Grain size } \\
\mathrm{D}(\mathrm{nm})\end{array}$ & $\begin{array}{c}\mathrm{d} \text {-spacing } \\
(\mathrm{nm})\end{array}$ & $\begin{array}{c}\text { RMS Roughness } \\
(\mathrm{nm})\end{array}$ \\
\hline 230 & 3.32 & 15.096 & 0.262 & 12.1 \\
205 & 3.32 & 13.209 & 0.262 & 8.8 \\
105 & 3.31 & 13.519 & 0.262 & 5.1 \\
63 & 3.30 & 6.288 & 0.263 & 2.8 \\
30 & - & - & - & 1.3 \\
\hline
\end{tabular}

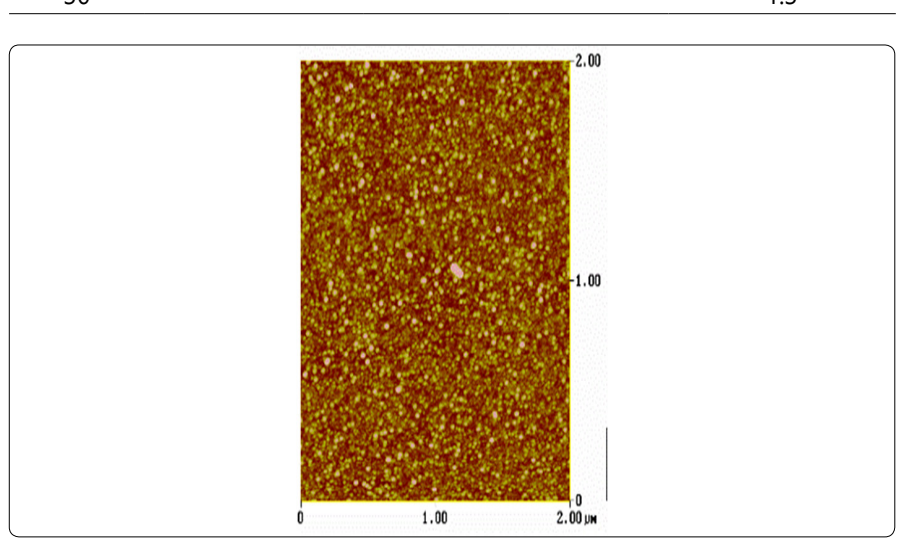

Figure 1(a). AFM images of $\mathrm{ZnO}$ films with thickness $30 \mathrm{~nm}$

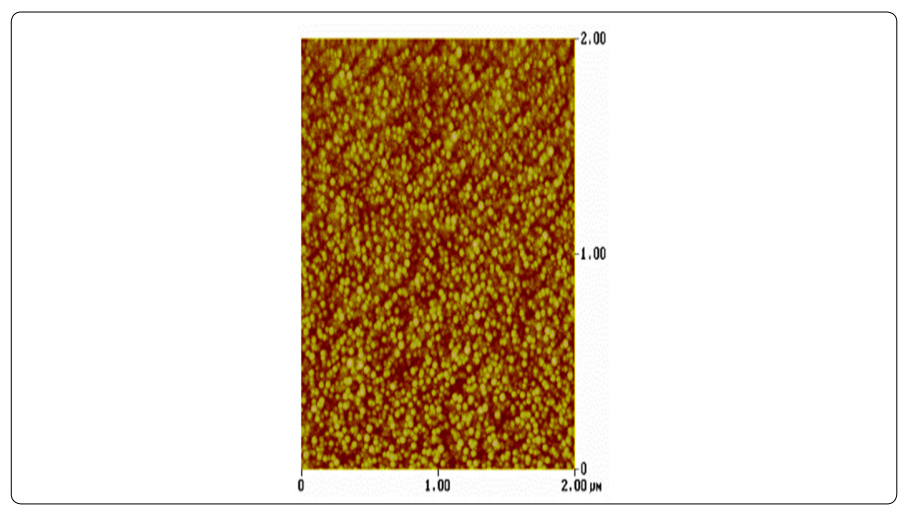

Figure 1(b). AFM images of ZnO films with thickness $63 \mathrm{~nm}$

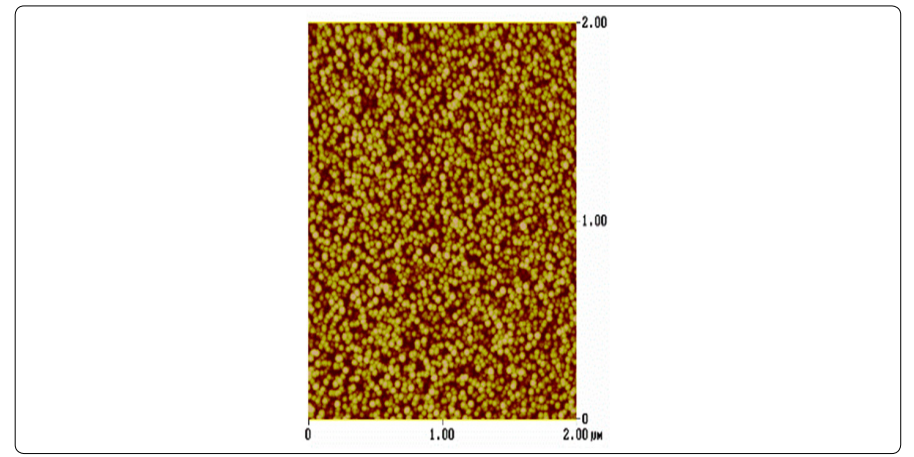

Figure 1(c). AFM images of $\mathrm{ZnO}$ films with thickness $105 \mathrm{~nm}$

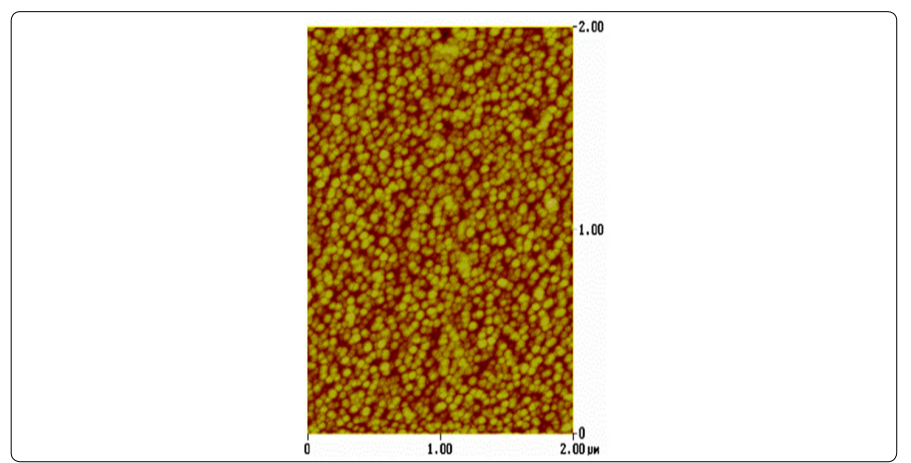

Figure 1(d). AFM images of ZnO films with thickness $205 \mathrm{~nm}$

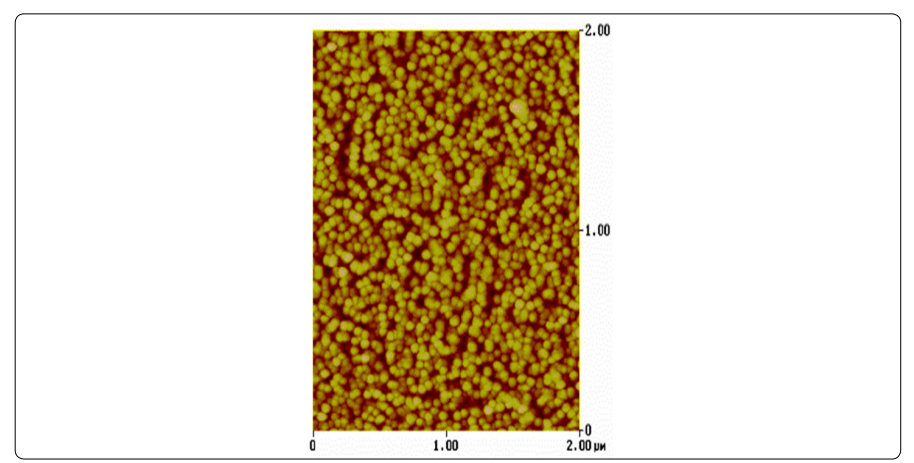

Figure 1(e). AFM images of $\mathrm{ZnO}$ films with thickness $230 \mathrm{~nm}$

The XRD patterns of the $\mathrm{ZnO}$ films shown in Fig. 2 were identified as the hexagonal wurtzite zinc oxide, (Joint Committee on Powder Diffraction Standards (JCPDS) card no. 36: 1451) with no impurities. It was apparent that as the thickness increases, the peak intensity at $2 \theta \approx 34.4^{\circ}$ corresponding to (002) plane increases, whereas its width decreases. This was an indication that the films were growing preferentially along the c-axis orientation, vertical to the substrate, a general observation for sputtered $\mathrm{ZnO}$ films [24] [25]. Higher crystallinity was obtained for thicker samples while the film with the thickness of $30 \mathrm{~nm}$ been regarded amorphous as diffraction peaks were missing. 


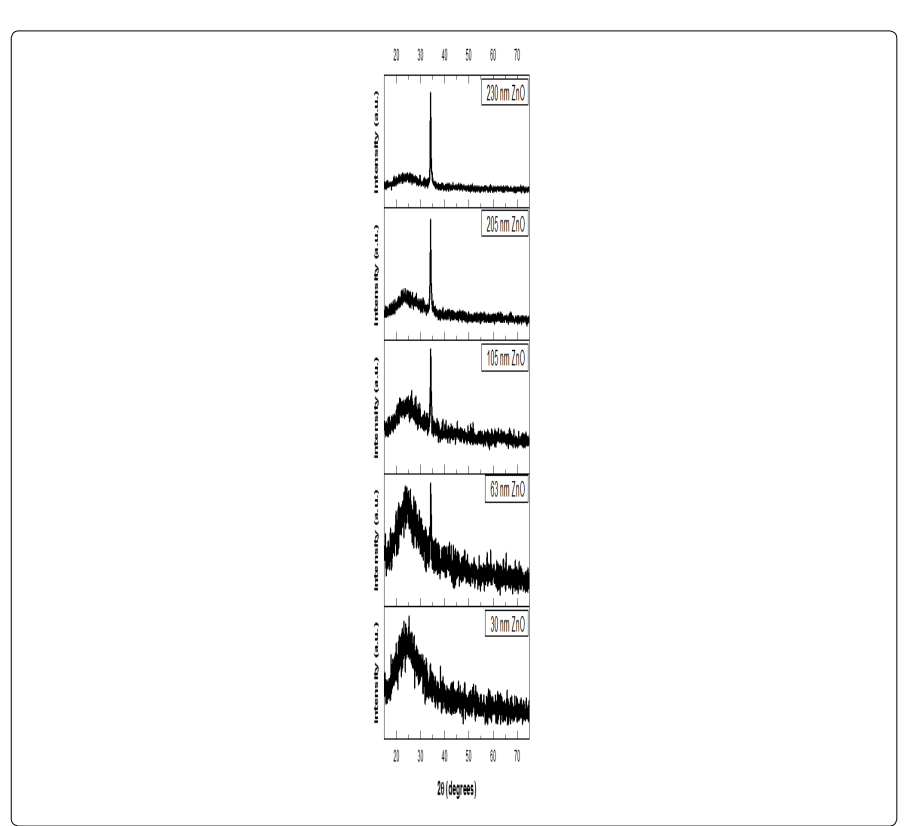

Figure 2. XRD pattern of $\mathrm{ZnO}$ thin film deposited at different thicknesses

Fig. 3 depicts the optical transmittance spectra, $T(\%)$, of ZnO films deposited onto Corning glass substrates. It can be seen that all films exhibited a high transparency over $80 \%$, in the visible and NIR regions, which is in line with other studies [24] [26]. The optical band gap of $\mathrm{ZnO}$ was found to be in the range of 3.32 to $3.30 \mathrm{eV}$ as shown in the insets. The variations of structural and optical characteristics with thickness are being presented in Table 1. It is obvious that both energy band gap and $d$-spacing remain almost constant, while the grain size increases with thickness, confirming other studies [21] [27].
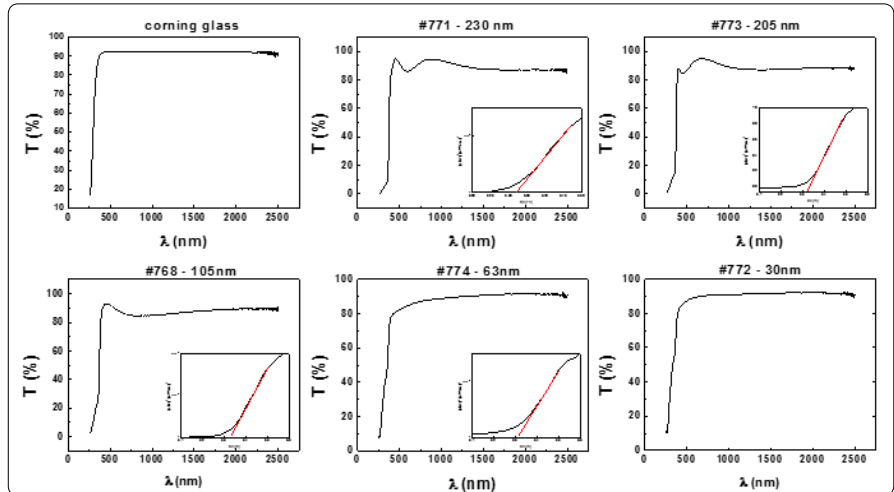

Figure 3. Transmittance spectra of $\mathrm{ZnO}$ thin films. Inset: Variation of $(a h v)^{2}$ vs. (hv)

\section{Sensing properties \\ Conductometric technique}

Monitoring of conductivity at different ozone concentration levels provides information on the sensing properties of the films under test. Fig. 4 illustrates typical cycles of a consecutive photoreduction-oxidation process for the $\mathrm{ZnO}$ film with a thickness of $230 \mathrm{~nm}$. The first oxidation process took place in the presence of synthetic air $\left(20 \% \mathrm{O}_{2}, 80 \% \mathrm{~N}_{2}\right)$ which was used as the reference signal while the remaining oxidation processes were carried out under different ozone concentrations. The duration of each photoreduction process was 10 min while each oxidation process lasted $15 \mathrm{~min}$.

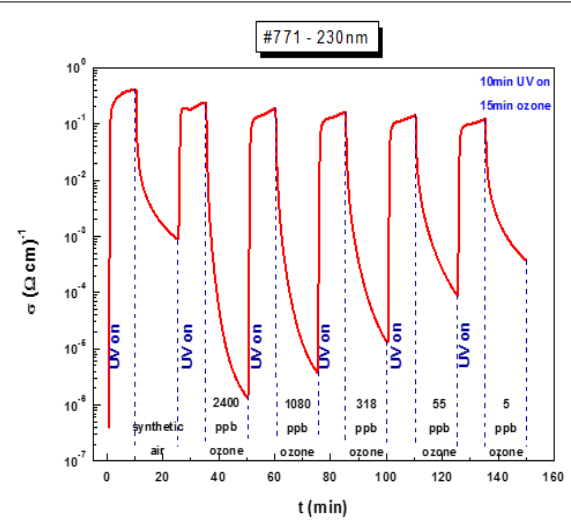

Figure 4. Photoreduction-oxidation cycles of $\mathrm{ZnO}$ film $230 \mathrm{~nm}$

Results have shown that subject to ozone concentration exposures ranging between 2400 to $5 \mathrm{ppb}$, the conductivity varied between $10^{-6}$ to $10^{-1}(\Omega \mathrm{cm})^{-1}$ depending on the film, resulting to 3-5 orders of magnitude changes, making this film a highly sensitive ozone sensor in line with past reports on sputtered $\mathrm{ZnO}$ thin films [22] [27]. It should be noted that, when chemical techniques are used for the growth of $\mathrm{ZnO}$, namely spray pyrolysis [8], sol gel dip coating [28], chemical vapor deposition (CVD) [29], and aqueous solution method [30], a much lower change in conductivity ( 1 and 3 orders of magnitude) at various oxidizing gases has been reported. It is important to note that in the present study for each photoreduction cycle the maximum conductivity values remain almost constant and independent of subsequent ozone concentration exposure, denoting that the overall number of electrons created during the photoreduction cycle remains almost unaffected. However, the sensing responses during the different oxidation cycles were found to be a function of the $\mathrm{O}_{3}$ concentration, thus the responses recorded were distinctively decreasing with decreasing $\mathrm{O}_{3}$ exposure level which is the desirable result as far as sensing capability is concerned. Furthermore, a sensing element should be able to distinguish among the different gas concentrations present, exhibiting different response rates. Fig. 5 depicts the normalized exponential decay curves obtained from the above oxidation processes. The slope of each curve represents the ability of the material under test to sense different ozone concentration levels with adequate resolution. It was found that this was achieved from the first minute of exposure even for the lowest concentration of $5 \mathrm{ppb}$.

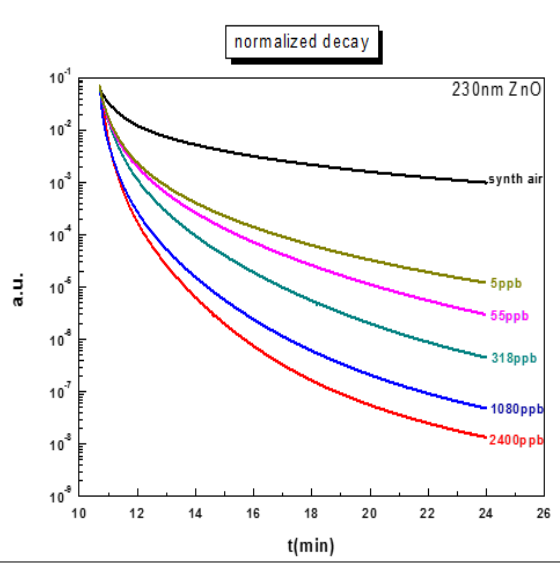

Figure 5. Normalized exponential decay curves of $230 \mathrm{~nm}$ thick $\mathrm{ZnO}$ sensor 
Other films with similar thicknesses exhibited equivalent results (e.g. the $205 \mathrm{~nm}$ film). However, for films with lower thicknesses, as in the case of the $105 \mathrm{~nm}$ sample (Fig. 6, a) and less, although the response rate was satisfactory ( 3 orders of magnitude), the decay curves to different ozone levels concentrations could not be adequately distinguished, exhibiting poor resolution (Fig. 6, b). This behaviour was attributed to the specific growth conditions and the corresponding film crystalline structure, and maybe associated with the depleted area created following the photoreduction cycles.

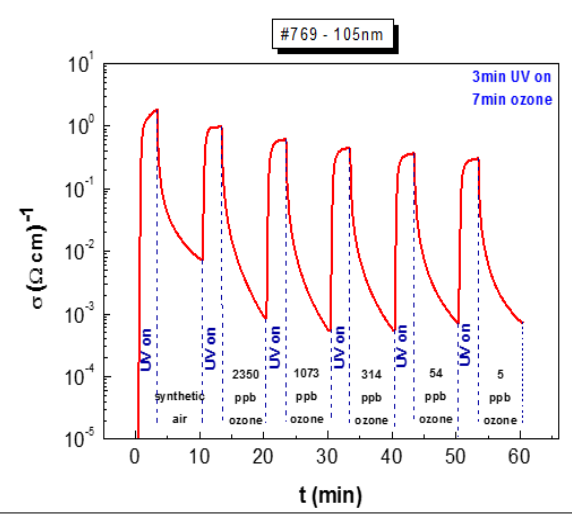

Figure 6(a). Photoreduction-oxidation cycles of $\mathrm{ZnO} 105 \mathrm{~nm}$

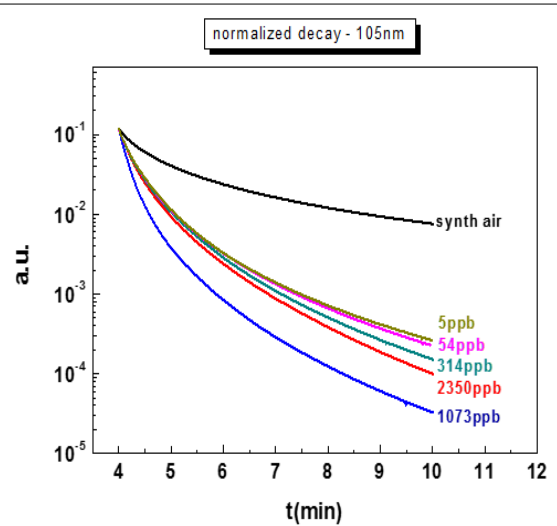

Figure 6(b). Normalized exponential decay curves of $105 \mathrm{~nm}$

It is widely accepted that for the metal oxide gas sensing elements, during the photoreduction process, oxygen bonds are breaking leading to the creation of oxygen vacancies in a process by which two electrons are being released for each missing oxygen [31]. In the subsequent process of oxidation, the sensitivity of the sensing elements seems to be subject to the presence of the oxidizing gas $\left(\mathrm{O}_{3}\right)$ i.e. decreases with the decrease of ozone concentration levels while the response is higher at higher concentration levels [28] [29]. The recorded gradual drop in the observed maximum conductivity levels after the photoreduction process, particularly for the thinner films may be attributed to the fact that the oxygen vacancies in the photoreduced film are not fully occupied by the oxygen atoms of the ozone molecules during the oxidation process. Therefore by decreasing the ozone concentration level the response obtained, after the photoreduction, was lower. For the thinner samples in particular, the developed oxygen vacancies during photoreduction may initially be occupied completely from the first ozone concentration levels during the oxidation process thus leading to a fast saturation. Such a process already observed in the past, seems to be reproducible and consistent with each and every photoreduction-oxidation test cycle [8].

The sensitivity of films with different thickness was calculated as the ratio of the maximum to minimum conductivity values, $\left(S=\sigma_{\max } / \sigma_{\min }\right)$ [1] and their normalized relative sensitivity responses as a function of ozone concentration levels are presented in Fig. 7. It is apparent that for ozone concentrations below 500 ppb the sensitivity of almost all sputtered films remains constant and any variations are within one order of magnitude only, independent of the film thickness. This denotes the films inability to safely distinguish among $\mathrm{O}_{3}$ concentrations in this low volume range. On the other hand, for ozone concentrations above $500 \mathrm{ppb}$ and thickness above $200 \mathrm{~nm}$ the responses are obviously much larger, implying that although the $\mathrm{ZnO}$ sensing materials can detect ultra low ozone levels of the order of a few ppb, they lack the required resolution at concentrations below 500 ppb, exhibiting a much better ability to distinguish between higher ozone levels. Comparing the present results with those existing in the literature, it is confirmed that the conductivity and gas sensitivity increase with the increase of the crystallite size [32], and consequently with the increase of the thickness.

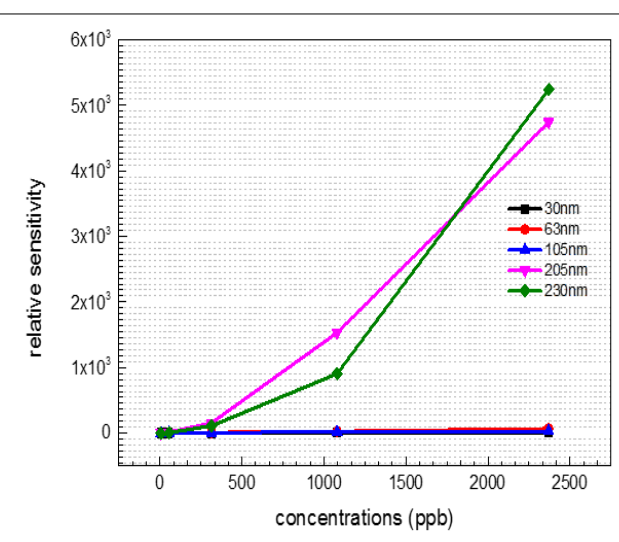

Figure 7. Normalized relative Sensitivity responses as a function of concentration for all thicknesses

\section{SAW technique}

Contrasting the above results with the SAW technique, two major interactions i.e. the mass loading and acousto-electric effects were studied utilizing commercially available SAW filters. The first effect is associated with a noticeable frequency shift originating from mass loading. In particular, due to the deposition of a thin $\mathrm{ZnO}$ film, used as the sensitive layer, on top of the SAW filter, a small shift towards lower frequencies is being recorded, however, with no noticeable changes to the filter attenuation signal [33]. This happens, because any increase in the kinetic energy density (due to the wave movement on the surface) results in a decrease in the wave velocity, hence in the signal transmission frequency $(v=u / \lambda$, where $v$ : frequency, $\lambda$ : wavelength and $u$ : velocity). Following this initial frequency shift which should remain constant (its value is considered as the central reference frequency for detecting subsequent acoustic wave velocity variations) any surface perturbations as a result of a photoreduction and oxidation processes on the sensing film surface are recorded with distinct clarity. 
In order to examine the acousto-electric behavior of the SAW filters utilizing a thin sensing film of $\mathrm{ZnO}$ ( $\mathrm{ZnO} / \mathrm{SAW}$ ) on top, and the resulting frequency shifts as a response to surface perturbations, filters with a nominal central operating frequency of $930 \mathrm{MHz}$ were selected. Their responses as a function of different $\mathrm{ZnO}$ thicknesses were tested towards ozone with varying concentrations at room temperature conditions. Fig. 8, a-c depicts typical centre frequency variations towards consecutive photoreduction-oxidation cycles against four different ozone concentrations. Every cycle lasted $15 \mathrm{~min}$, of which 5 min were dedicated for the photoreduction process while $10 \mathrm{~min}$ was the duration of each oxidation process. The studied film thicknesses of the $\mathrm{ZnO}$ films were limited to less than $100 \mathrm{~nm}$ as any greater thickness imposed a significant mass loading effect, therefore additional stress which subsequently introduced an operating malfunction on the sensitivity of the selected SAW filters resulting in significant deviations from their normal operational characteristics.

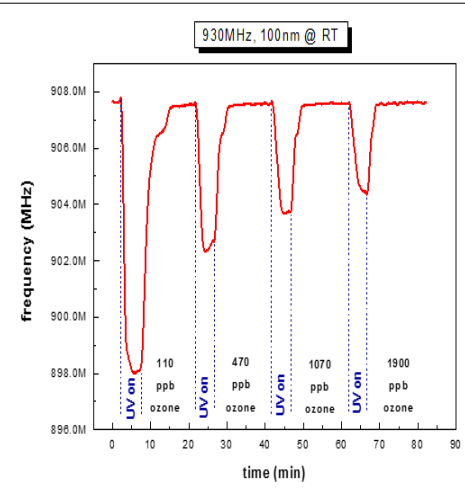

Figure 8(a). $930 \mathrm{MHz}$ SAW filter response towards different $\mathrm{O}_{3}$ concentrations at RT for three different $\mathrm{ZnO}$ thicknesses $105 \mathrm{~nm}$

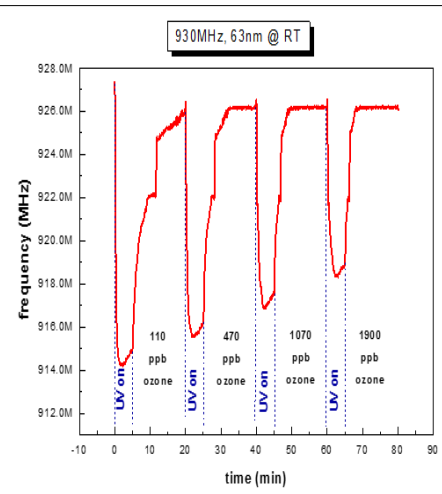

Figure 8(b). $930 \mathrm{MHz}$ SAW filter response towards different $\mathrm{O}_{3}$ concentrations at RT for three different $\mathrm{ZnO}$ thicknesses $63 \mathrm{~nm}$

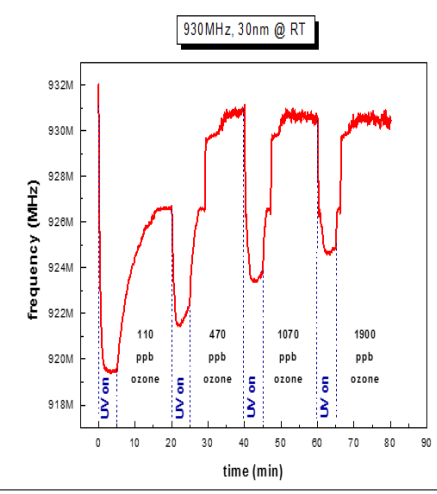

Figure 8(c). $930 \mathrm{MHz}$ SAW filter response towards different $\mathrm{O}_{3}$ concentrations at RT for three different $\mathrm{ZnO}$ thicknesses $30 \mathrm{~nm}$
Results from $\mathrm{ZnO}$ films with thicknesses less than $100 \mathrm{~nm}$ revealed a decrease in the filter's centre frequency under UV illumination and a subsequent frequency increase under ozone exposure during a photoreduction-oxidation cycle. This behavior is in good agreement with published works [27] [32] [34] where other oxidizing gases such as $\mathrm{NO}_{2}$ were used. Analysing the present results in more detail, we observed frequency response of the order of 8-9 $\mathrm{MHz}$ in the commercial SAW filters following the deposition of the $\mathrm{ZnO}$ sensitive layer with thickness ranging between $30-100 \mathrm{~nm}$. This was due to the mass loading effect and is justified by the fact that the sensing material $(\mathrm{ZnO})$ is highly insulating i.e. induces no inherent acousto-electric effects. In a study presented by $B$. Raj and co-workers in 2013 [35] ZnO/SAW deposited by sputtering was compared with other metal oxides $\left(\mathrm{SnO}_{2}, \mathrm{TeO}_{2}\right.$ and $\mathrm{TiO}_{2}$ ) and they found that $\mathrm{ZnO}$, in a parametric study on the thickness dependence, exhibited higher responses under a selected reducing gas (ammonia) exposure. These results are in a good agreement with the present work during which we achieved to obtain detectable responses towards ultralower $\mathrm{O}_{3}$ concentrations at a range of low film thickness. Alternative studies on $\mathrm{ZnO}$ nanoparticles deposited by spin coating onto SAW devices by Phan and co-workers in 2012 [36] on hydrogen sensing applications concluded that a layered SAW structure may exhibit a frequency shift at low $\mathrm{H}_{2}$ concentration at room temperature with good repeatability and stability. However in this study the maximum measured shift was in the order of $\mathrm{kHz}$. As opposed to our results obtained at RT, their good response was due to the postannealing process and consequently improvement of the films crystallinity properties. In the present study, following subsequent analysis applying cycles of photoreduction and oxidation, a noticeable drift of the base line frequency was recorded, denoting an increasing memory effect during the initial stages of the oxidation state particularly for the lower thickness films and at low $\mathrm{O}_{3}$ concentrations. It is apparent that as the gas concentration increases the recorded frequency response increases. This may be attributed to the fact that as ozone is induced into the test chamber, an increased coverage of the vacancies sites is induced, resulting to an excess of oxygen presence on the surface sites. Fig. 9 depicts a complete single photoreduction-oxidation cycle of the film with thickness $30 \mathrm{~nm}$ which is subjected to a more detailed subsequent analysis hereby. When the photoreduction process starts with UV light on, the process competes with an inherent oxidation process due to the $\mathrm{O}_{3}$ formation by the UV radiation on the poor vacuum environment (10-2 mbar) of the test chamber. (Fig. 9, region A1) the analysis of the obtained results, it is observed that after several seconds and while the samples are still illuminated by the UV lamp, a frequency increase takes place which is attributed to the fact that exposure of the film to the UV light in an environment that there are oxygen molecules, the photoreduction is losing to an oxidation process that takes place while the films is under UV illumination [37] (Fig. 9, region A2). Further analysis revealed that in the absence of UV light and simultaneous ozone introduction into the test chamber, the main oxidation 
process initiates. During this stage, a two-step procedure seems to be involved, one attributed purely to an electric current variation (reduction) due to the capture of free electrons created during the UV photoreduction process and another, most probably associated with mechanical induced changes possibly attributed to the piezoelectric substrate material. This effect was more intense on filters with thin sensing layers.

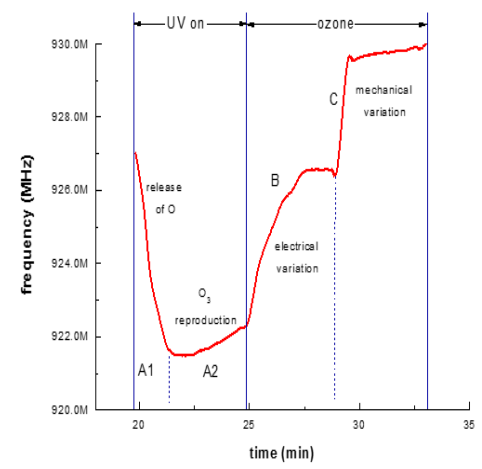

Figure 9. Detailed representation of a single photoreduction-oxidation procedure through the SAW device of Fig. $8 \mathrm{C}$

In more detail, the region marked as B in Fig. 9 represents the first step of the oxidation process and can be attributed to the SAW acousto-electric effect. This sensing behaviour corresponds exactly to the earlier results from the conductometric response already analysed and are attributed to surface phenomena. It must be emphasized, that this step ends at the same frequency value and is dependent of the gas concentration, indicating that it is a purely surface transport phenomenon as confirmed by conductometric measurements. The second step of the oxidation process, marked as C (Fig. 9), is probably related to a mechanical phenomenon relevant to the nature of the SAW transducer used as substrate material rather than a direct acousto-electric effect. Supporting evidence on this argument is the fact that no such behaviour has ever been reporting on metal oxide sensing layers deposited on other rigid or flexible substrates. Therefore, it is believed that this is most likely a phenomenon concerning the mechanical properties of the used substrate i.e. it is directly related to the elasticity of the piezoelectric substrate. Such a change in a SAW filter mechanical properties may be attributed to the interaction of ozone with the SAW structure itself that leads to changes to its elastic (Young's) modulus. In general piezoelectric materials couple electrical and mechanical effects along with thermal effects therefore they are sensitive to a number of parameters. These are usually described by the following relations [19]:

$$
\begin{aligned}
& T=c^{E} S-e E \\
& D=\varepsilon^{S} E+e S
\end{aligned}
$$

in which $\mathrm{T}$ is the mechanical stress $\left(\mathrm{Nm}^{-2}\right), \mathrm{S}$ is the stiffness with constant electric field, $\mathrm{S}$ is the mechanical strain, $\mathrm{e}$ is the piezoelectric stress constant $\left(\mathrm{Cm}^{-2}\right), \mathrm{E}$ is the electric field $\left(\mathrm{Vm}^{-1}\right)$, $D$ is the electric displacement $\left(\mathrm{Cm}^{-2}\right)$, and $\varepsilon$ is the permittivity $\left(\mathrm{CV}^{-1} \mathrm{~m}^{-1}\right)$ at constant strain [19]. From the above relations and experimental data it becomes apparent that the introduction of ozone causes electrical variations (represented in B region) which are leading to a decrease in conductivity; this is something also confirmed by the above conductometric results (Fig. $4 \&$ Fig. 6a). While still under $\mathrm{O}_{3}$ exposure (region B) the free electron capture process is getting saturated (plateau at the end of region $B$ ) and any electrical variations are terminated leading to a constant electric field presence. If this is true, the second term in the right-hand side of Eq.1 remains constant denoting a relation between stress and strain (Young's modulus). Therefore, ozone introduction may result to mechanical variations through elasticity changes of the SAW filter substrate material which in turn induce additional stress $(T)$. These variations may then lead to an increase in the acoustic wave velocity and hence the propagating frequency (see region marked as $C$ ) which is also confirmed by similar reports published by others [35] [38].

In conclusion it is suggested that these frequency changes may be attributed to induced mechanical variations of the SAW filter posed by the presence of ozone atmosphere, a result which requires further verification through additional future work.

Summarizing the results of the present work, one may conclude that $\mathrm{ZnO}$ films grown by $\mathrm{DC}$ sputtering are very promising candidates for ozone sensing applications capable to detect concentrations of the order of a few ppb at room temperature. Although, the use of SAW filters induces additional implications on the signal responses which combined with the fact that there is no recorded improvement in the ozone detection limits compared to the single conductometric technique renders the SAW, as a technique itself, applicable only in dedicated applications for which integration of a SAW filter may be regarded more advantageous than a simple conductometric set-up.

\section{Conclusion}

The ozone sensing properties of zinc oxide utilizing a conductometric set-up as bench-mark for surface acoustic wave gas sensors were studied. All films, with the exception of the 30 $\mathrm{nm}$ thick film were polycrystalline as revealed from the XRD analysis, with the preferential orientation along the c-axis in the (002) plane. They also exhibited a high transparency $(>80 \%)$ in the visible region. AFM images of their surfaces revealed that roughness increases along with thickness. Sputtered films with different thicknesses have showed conductivity changes of up to 5 orders of magnitude during a photoreduction-oxidation cycle. As far as SAW results are concerned, the study has shown that, due to the mass loading effect, a decrease of the centre frequency takes place along with the deposited $\mathrm{ZnO}$ sensitive layer. The acousto-electric response appeared to be the dominant effect regarding electrical conductivity variations. The acousto-electric effect revealed a good response of the sensing element with a frequency shift of a few MHz. SAW filter sensing analysis revealed that the oxidation process is mainly a two-step procedure. The first step was attributed solely to the induced electrical variation while the second to the mechanical variations, possibly originating from the substrate piezoelectric properties. Comparing the conductometric with the SAW technique one may conclude that the first technique is preferable for an accurate account of ultra-low $\mathrm{O}_{3}$ concentrations. 


\section{Acknowledgments}

The support through the FP7-Regpot-2012-2013-1 project under grant agreement No 316165 is highly appreciated.

\section{References}

1. Bender $M$, Fortunato EP, Nunes $A$, et al. Highly sensitive $\mathrm{ZnO}$ ozone detectors at room temperature. Japanese J Appl Physics, Part 2 Lett. 2003; 42 (4 B): 2-5. doi: 10.1143/JJAP.42.L435

2. Look DC. Recent advances in $\mathrm{ZnO}$ materials and devices. Mater Sci Eng $B$ Solid-State Mater Adv Technol. 2001; 80(1-3): 383-87. doi: 10.1016/ S0921-5107(00)00604-8

3. Suchea M, Christoulakis S, Moschovis K, Katsarakis N, Kiriakidis G. ZnO transparent thin films for gas sensor applications. Thin Solid Films. 2006; 515(2): 551-54. doi: 10.1016/j.tsf.2005.12.295

4. Kortidis I, Moschovis K, Mahmoud FA, Kiriakidis G. Structural analysis of aerosol spray pyrolysis $\mathrm{ZnO}$ films exhibiting ultra low ozone detection limits at room temperature. Thin Solid Films. 2009; 518(4): 1208-13. doi: 10.1016/j.tsf.2009.08.018

5. Lee GH, Kawazoe T, Ohtsu M. Difference in optical bandgap between zinc-blende and wurtzite $\mathrm{ZnO}$ structure formed on sapphire (0001) substrate. Solid State Commun. 2002; 124(5): 163-65. doi: 10.1016/ S0038-1098(02)00537-9

6. Martinez-Perez L, Munoz-Aguirre N, Munoz-Aguirre S, Zelaya-Angel O. Nanometric structures of highly oriented zinc blende $\mathrm{ZnO}$ thin films. Mater Lett. 2015; 139: 63-65. doi: 10.1016/j.matlet.2014.10.054

7. Özgür Ü, Alivov $\mathrm{YI}$, Liu CA, et al. A comprehensive review of $\mathrm{ZnO}$ materials and devices. J Appl Phys. 2005; 98(4): 1-103. doi: 10.1063/1.1992666

8. Bender M, Gagaoudakis E, Douloufakis E, et al. Production and characterization of zinc oxide thin films for room temperature ozone sensing. Thin Solid Films. 2002; 418(1): 45-50. doi: 10.1016/S0040-6090(02)00588-6

9. Ippolito SJ, Kandasamy S, Kalantar-Zadeh K, et al. Highly Sensitive Layered $\mathrm{ZnO} / \mathrm{LiNbO}_{3}$ SAW Device with InOx Selective Layer for $\mathrm{NO}_{2}$ and $\mathrm{H}_{2}$ Gas Sensing. Sensors and Actuators B: Chemical. 2005; 111-112: 207-12. doi: 10.1016/j.aca.2012.05.048

10. Özgür Ü, Hofstetter D, Morkoç H. ZnO Devices and Applications: A Review of Current Status and Future Prospects. Proc IEEE. 2010; 98(7): 1255-68. doi: 10.1109/JPROC.2010.2044550

11. EPA. NAAQS Table|Criteria Air Pollutants. https://www.epa.gov/criteriaair-pollutants/naaqs-table. Published 2008.

12. Atashbar $M Z$, Gong B, Sun HT, Wlodarski W, Lamb R. Investigation on ozone-sensitive In2O3 thin films. Thin Solid Films. 1999; 354(1): 222-26. doi: 10.1016/S0040-6090(99)00405-8

13. Thirumalairajan $S$, Mastelaro VR. A novel organic pollutants gas sensing material p-type CuAlO2 microsphere constituted of nanoparticles for environmental remediation. Sensors Actuators, B Chem. 2016; 223: 138-48. doi: 10.1016/j.snb.2015.09.092

14. Wu CH, Jiang GJ, Chang KW, Lin CW, Chen KL. Highly sensitive amorphous In-Ga-Zn-O films for ppb-level ozone sensing: Effects of deposition temperature. Sensors Actuators, B Chem. 2015; 211: 354-58. doi: 10.1016/j.snb.2015.01.048

15. Kang $B S$, Heo $Y W$, Tien $L C$, et al. Hydrogen and ozone gas sensing using multiple ZnO nanorods. Appl Phys A. 2004; 80(5): 1029-32. doi: 10.1007/ s00339-004-3098-8

16. Hoummady M, Campitelli A, Wlodarski W. Acoustic wave sensors: design, sensing mechanisms and applications. Smart Mater Struct. 1997; 6(6): 647-57. doi: 10.1088/0964-1726/6/6/001

17. Drafts B. Acoustic Wave Technology Sensors. IEEE Trans Microw Theory Tech. 2001; 49(4): 795-802.

18. Kirschner J. Surface Acoustic Wave Sensors (SAWS): Design for Application. Microelectromechanical Syst. 2010: 1-11.
19. Ballantine DS, Martin SJ, Ricco AJ, Ricco AJ, Frye GC, Wohltjen H, White RM, Zellers ET. Acoustic Wave Sensors.1997.

20. Papadaki $\mathrm{D}$, Foteinis $\mathrm{S}$, Mhlongo $\mathrm{GH}$, et al. Life cycle assessment of facile microwave-assisted zinc oxide ( $\mathrm{ZnO})$ nanostructures. Sci Total Environ. 2017; 586: 565-75. doi: 10.1016/j.scitotenv.2017.02.019

21. Lin S, Huang J, Lii D. Effect of substrate temperature on the properties of Ti-doped $\mathrm{ZnO}$ films by simultaneous if and dc magnetron sputtering. MaterChem Phys. 2005; 90:22-30. doi: 10.1016/j.matchemphys.2004.08.040

22. Ahmadipour M, Ain MF, Ahmad ZA. Effect of thickness on surface morphology, optical and humidity sensing properties of RF magnetron sputtered CCTO thin films. Appl Surf Sci. 2016; 385: 182-90. doi: 10.1016/j.apsusc.2016.05.098

23. Bedia A, Bedia FZ, Aillerie $\mathrm{M}$, et al. Optical, electrical and structural properties of nano-pyramidal $\mathrm{ZnO}$ films grown on glass substrate by spray pyrolysis technique. Opt Mater (Amst). 2014; 36(7): 1123-30. doi: 10.1016/j.optmat.2014.02.012

24. Tseng JY, Chen YT, Lin CJ, et al. The optical, electrical, and nanomechanical properties of ZnO thin films. Opt - Int J Light Electron Opt. 2015; 126(21): 3263-66. doi: 10.1016/j.jijleo.2015.07.126

25. Musat V, Rego AM, Monteiro R, Fortunato E. Microstructure and gassensing properties of sol-gel $\mathrm{ZnO}$ thin films. Thin Solid Films. 2008; 516(7): 1512-15. doi: 10.1016/j.tsf.2007.07.198

26. Jin M, Ying LS. Preparation of $\mathrm{ZnO}$ films by reactive evaporation. Thin Solid Films. 1994; 237: 16-18.

27. Kiriakidis G, Moschovis K, Kortidis I, Binas V. Ultra-low gas sensing utilizing metal oxide thin films. Vacuum. 2012; 86(5): 495-506. doi: 10.1016/j.vacuum.2011.10.013

28. Cheng XL, Zhao H, Huo LH, Gao S, Zhao JG. ZnO nanoparticulate thin film: Preparation, characterization and gas-sensing property. Sensors Actuators, B Chem. 2004; 102(2): 248-52. doi: 10.1016/j.snb.2004.04.080

29. Shao $C$, Chang $Y$, Long $Y$. High performance of nanostructured $\mathrm{ZnO}$ film gas sensor at room temperature. Sensors Actuators, B Chem. 2014; 204: 666-72. doi: 10.1016/j.snb.2014.08.003

30. Huang J, Wu Y, Gu C, Zhai M, Sun Y, Liu J. Fabrication and gas-sensing properties of hierarchically porous $\mathrm{ZnO}$ architectures. Sensors Actuators, B Chem. 2011; 155(1): 126-33. doi: 10.1016/j.snb.2010.11.036

31. de Lacy Costello BPJ, Ewen RJ, Ratcliffe NM, Richards M. Highly sensitive room temperature sensors based on the UV-LED activation of zinc oxide nanoparticles. Sensors Actuators, B Chem. 2008; 134(2): 945-52. doi: 10.1016/j.snb.2008.06.055

32. Ippolito SJ, Kandasamy S, Kalantar-Zadeh K, et al. Highly sensitive layered $\mathrm{ZnO} / \mathrm{LiNbO} 3 \mathrm{SAW}$ device with $\mathrm{InO} \times$ selective layer for $\mathrm{NO} 2$ and H2 gas sensing. Sensors Actuators, B Chem. 2005; 111-112(SUPPL.): $207-$ 12. doi: $10.1016 / j . s n b .2005 .07 .046$

33. Cheeke JDN, Wang Z. Acoustic wave gas sensors. Sensors Actuators $B$ Chem. 1999; 59(2-3): 146-53. doi: 10.1016/S0925-4005(99)00212-9

34. Matatagui $\mathrm{D}$, Fernandez MJ, Fontecha JL, et al. Propagation of acoustic waves in metal oxide nanoparticle layers with catalytic metals for selective gas detection. Sensors Actuators B Chem. 2015; 217: 65-71. doi: 10.1016/j.snb.2014.12.076

35. Bhasker Raj V, Singh H, Nimal AT, Tomar M, Sharma MU, Gupta V. Effect of metal oxide sensing layers on the distinct detection of ammonia using surface acoustic wave (SAW) sensors. Sensors Actuators, B Chem. 2013, 187: 563-73. doi: 10.1016/j.snb.2013.04.063

36. Phan DT, Chung GS. Surface acoustic wave hydrogen sensors based on $\mathrm{ZnO}$ nanoparticles incorporated with a Pt catalyst. Sensors Actuators $B$ Chem. 2012; 161(1): 341-48. doi: 10.1016/j.snb.2011.10.042

37. Martins $R$, Fortunato $E$, Nunes $P$, et al. Zinc oxide as an ozone sensor. J Appl Phys. 2004; 96(3): 1398-108. doi: 10.1063/1.1765864

38. Jakubik WP. Surface acoustic wave-based gas sensors. Thin Solid Films. 2011; 520(3): 986-93. doi: 10.1016/j.tsf.2011.04.174 